Research article

\title{
A model for the proteolytic regulation of LpxC in the lipopolysaccharide pathway of Escherichia coli
}

\author{
Akintunde Emiola ${ }^{\mathrm{a}, *}$, Paolo Falcarin ${ }^{\mathrm{b}}$, Joanne Tocher ${ }^{\mathrm{a}}$, John George ${ }^{\mathrm{a}}$ \\ a School of Health, Sports and Bioscience, University of East London, Stratford Campus, London E15 4LZ, United Kingdom \\ ${ }^{\mathrm{b}}$ School of Architecture, Computing and Engineering, University of East London, Docklands Campus, London E16 2RD, United Kingdom
}

\section{A R T I C L E I N F O}

\section{Article history:}

Received 3 May 2013

Received in revised form 3 June 2013

Accepted 4 June 2013

\section{Keywords:}

Metabolic pathway

Lipid A

Simulation

FtsH cleavage

\begin{abstract}
A B S T R A C T
Lipopolysaccharide (LPS) is an essential structural component found in Gram-negative bacteria. The molecule is comprised of a highly conserved lipid A and a variable outer core consisting of various sugars. LPS plays important roles in membrane stability in the bacterial cell and is also a potent activator of the human immune system. Despite its obvious importance, little is understood regarding the regulation of the individual enzymes involved or the pathway as a whole. LpxA and LpxC catalyze the first two steps in the LPS pathway. The reaction catalyzed by LpxA possesses a highly unfavourable equilibrium constant with no evidence of coupling to an energetically favourable reaction. In our model the presence of the second enzyme LpxC was sufficient to abate this unfavourable reaction and confirming previous studies suggesting that this reaction is the first committed step in LPS synthesis. It is believed that the protease FtsH regulates LpxC activity via cleavage. It is also suspected that the activity of FtsH is regulated by a metabolite produced by the LPS pathway; however, it is not known which one. In order to investigate these mechanisms, we obtained kinetic parameters from literature and developed estimates for other simulation parameters. Our simulations suggest that under modest increases in LpxC activity, FtsH is able to regulate the rate of product formation. However, under extreme increases in LpxC activities such as over-expression or asymmetrical cell division then FtsH activation may not be sufficient to regulate this first stage of synthesis.
\end{abstract}

(c) 2013 Elsevier Ltd. All rights reserved.

\section{Introduction}

Lipopolysaccharide (LPS) is an important structural component in the outer membrane of Gram-negative bacteria (Poxton, 1995). This molecule is involved in important biological properties such as immune evasion, membrane stabilization and attachment to various surfaces (Walker et al., 2004). LPS has three distinctive structural sections; lipid A, core oligosaccharide and O-antigen (Raetz and Whitfield, 2002). The lipid A component is essential for growth and its structure is relatively well conserved amongst the Gram-negatives (Raetz et al., 2009). Biochemical studies of the LPS pathway have proved difficult for a number of reasons. Firstly, some of the reactions occur within the cytoplasm whilst subsequent steps occur within the membrane (Raetz and Whitfield, 2002). Secondly, a number of these enzymes have been difficult to purify (Garrett et al., 1997) presumably due to stability problems and finally, the substrates and products of some of these reactions are difficult to assay for (Belunis and Raetz, 1992). Therefore, an in silico metabolic

\footnotetext{
* Corresponding author. Tel.: +44 7508582840.

E-mail addresses: u1114196@uel.ac.uk, tunsemi@yahoo.com (A. Emiola).
}

model of this pathway would prove extremely useful for a complete understanding of both the synthesis and regulation of LPS.

The first obligatory enzyme in the biosynthesis of lipid $A$ is UDP-N-acetylglucosamine acyltransferase (LpxA) (Anderson and Raetz, 1987). This enzyme is encoded for by the lpxA gene (Coleman and Raetz, 1988) and is responsible for the transfer of R-3hydroxyl chain to glucosamine 3-OH of UDP glucosamine from R-3-hydroxyacyl-acyl carrier protein (Anderson et al., 1985). Its substrate (UDP-N-acetylglucosamine) is located at a biosynthetic branchpoint which was previously unknown prior to the discovery of LpxA (Anderson and Raetz, 1987). This enables the substrate to be utilized either in the biosynthesis of lipid A or for the synthesis of peptidoglycan (Creuzenet et al., 2000). An interesting feature of the reaction catalyzed by LpxA is the thermodynamically unfavourable equilibrium constant of approximately 0.01 (Anderson et al., 1993). Such large unequilibriums exist for other enzymes such as creatine kinase which solve the energetics either by coupling the reaction to an energetically favourable one or by interacting directly with subsequent proteins (Shih and Whitesides, 1977). There is no evidence of either of these mechanisms occurring for this enzyme and to date there have been no studies aimed at examining the impact of this unfavourable reaction in context of subsequent lipid A metabolism. 
UDP-N-acetylglucosamine

$$
\text { LpxA }
$$

UDP-3-O-(3-hydroxymyristoyl)-N-acetylglucosamine

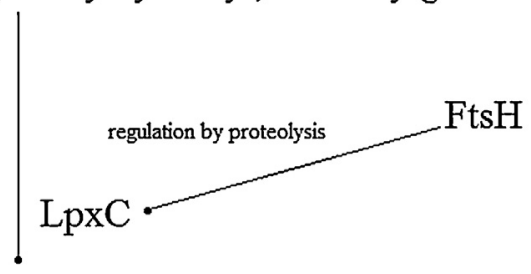

\section{UDP-3-O-(3-hydroxymyristoyl) glucosamine}

Fig. 1. A simple model for the first two steps in lipid A biosynthesis: LpxA and LpxC catalyze the first two steps in the Lipid A pathway and regulation of LpxC occurs via FtsH proteolytic cleavage.

UDP-3-O-N-acetylglucosamine deacetylase (LpxC) catalyzes the second reaction and due to the unfavourable LpxA reaction, it represents the first committed step in the biosynthesis of lipid A (Barb and Zhou, 2008). The reaction involves hydrolyzing UDP-3-O-(3-hydroxymyristoyl)-N-acetylglucosamine to UDP-3O-(3-hydroxymyristoyl) glucosamine and acetate (Young et al., 1995). It is known that very low levels of LpxC are detrimental to the cell viability (Schäkermann et al., 2013); however, very high levels of this enzyme also appear to be toxic to the organism presumably due to a build up of lipid A (Sorensen et al., 1996). Therefore, regulation of LpxC is crucial for microbial survival. In Escherichia coli, LpxC is regulated in a unique manner involving proteolytic cleavage of the enzyme by the protease FtsH (Ogura et al., 1999). The activity of the proteolysis is in turn thought to be dependent on the balance of phospholipid to lipopolysaccharide molecules (Schäkermann et al., 2013) but this has not been established experimentally. However, to date no studies have investigated the combined activities of the individual enzyme kinetic properties and subsequent regulatory mechanisms on the overall synthesis of LPS. Additionally, no mathematical models exist which would be capable of testing our current understanding based on published experimental research. Such a model could substantiate the proposed mechanism of lipid A synthesis as well as providing new insights into this complex and important microbial pathway.

Here we provide a model for the first two reactions of lipid A biosynthesis investigating the unfavourable reaction of LpxA and the subsequent committed step catalyzed by LpxC. Our model utilizes transcription/translation rates, enzymic rates and protein half lives/degradation rates to investigate the flow of substrates through this stage and interrogate the proposed regulatory mechanisms.

\section{Methods}

A simple model for the analysis of LpxA and LpxC in the biosynthesis of lipid A could be represented as shown in Fig. 1. In this paper, we provide a model for the first two reactions in the Lipid A biosynthetic pathway in $E$. coli for a single generation/doubling time.

The reactions were modelled by a simple enzymic reaction whereby, the enzyme E binds to the substrate $\mathrm{S}$, forming an enzyme-substrate complex ES, which then dissociates to produce the product $\mathrm{P}$ with release of free enzyme.

$\mathrm{E}+\mathrm{S} \underset{k_{-1}}{\stackrel{k_{1}}{\longrightarrow}} \mathrm{ES} \underset{k_{-2}}{\stackrel{k_{2}}{\longrightarrow}} \mathrm{P}+\mathrm{E}$

where $k_{1}$ is the rate constant of enzyme binding to the substrate to form the ES complex, $k_{-1}$ is the rate constant for dissociation from

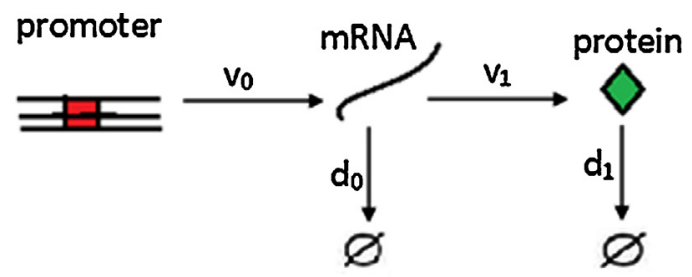

Fig. 2. A simple model of gene expression: A schematic representation of mRNA transcription and protein translation at a rate of $v_{0}$ and $v_{1}$ respectively. mRNA degrades at a rate $d_{0}$ whilst protein degradation occurs at a rate $d_{1}$.

the complex, $k_{2}$ is the catalytic rate constant and $k_{-2}$ the reverse rate constant for formation of the complex.

This simple enzymic model is not applicable to all reactions catalyzed by enzymes especially those involving more than one substrate. However, the reactions involved in the first two steps of lipid A biosynthesis involves more than one reactant. Prior kinetic studies on LpxA (Anderson et al., 1993) indicated that this enzyme followed a pseudo first-order process. Similarly, LpxC has as its second reactant $\mathrm{H}_{2} \mathrm{O}$, and given the abundance of water in the cell, this could also be modelled as a first order reaction.

\subsection{Estimation of transcription and translation rates of LpxA and LpxC}

A simple model for the transcription and translation of a gene can be represented as in Fig. 2 .

$v_{0}$ and $v_{1}$ are the rates of mRNA and protein synthesis respectively, while $d_{0}$ and $d_{1}$ represent the rate of mRNA and protein degradation respectively.

In our model, we made use of a generation time of $30 \mathrm{~min}$ (1800 s) for E. coli under optimum conditions (Plank and Harvey, 1979; Mackie, 2013). A mathematical model for estimating the number of proteins produced over a given period for a two-stage model of gene expression from information on available transcription/translation rates as well as mRNA and protein degradation rates have been proposed (Shahrezaei and Swain, 2008).

$$
\begin{aligned}
P_{n}(\tau)= & \frac{\Gamma(a+n)}{\Gamma(n+1) \Gamma(a)}\left(\frac{b}{1+b}\right)^{n}\left(\frac{1+b e^{-\tau}}{1+b}\right)^{a} \\
& \times{ }_{2} F_{1}\left(-n,-a, 1-a-n ; \frac{1+b}{e^{\tau}+b}\right)
\end{aligned}
$$

Eq. (1) gives the probability distribution of protein numbers $(n)$ as a function of time $t$ where $2 F 1(a, b, c ; z)$ is the hypergeometric function and $\Gamma$ represents the gamma function. Parameters $a$ correspond to the frequency of transcription burst relative to a protein lifetime $\left(v_{0} / d_{1}\right)$ while $b$ is the mean number of proteins produced per transcription burst $\left(v_{1} / d_{0}\right)$ and $\tau=t d_{1}$. The rate of protein production was modelled as a competition between ribosomes and RNAse E binding to the mRNA (McAdams and Arkin, 1997).

In our model, $v_{0}=$ Rate $_{\text {trans }}, v_{1}=$ Rate $_{\text {trsl }}, d_{0}=$ Rate $_{\text {degm }}$ and $d_{1}=$ Rate $_{\text {degp }}$

\subsubsection{Estimation of Rate trans, $_{\text {Rate }}$ trsl, Rate $_{\text {degm }}$ and Rate degp $_{\text {in }}$ LpxA}

It has been stated that the average number of transcription bursts of a gene per generation time for $E$. coli is between 5 and 10 (Mackie, 2013; Golding et al., 2005). However, since enzymes involved in LPS biosynthesis are constitutive (Raetz et al., 2009), the transcription burst value for LpxA is likely to be in the upper limit. As a result, our model assumes that LpxA transcription burst number would be about 7 times per generation time. We therefore assigned Rate trans $_{\text {to }}$ to $7 / 1800 \mathrm{~s}^{-1}$. The half life of some $E$. coli mRNAs have been analyzed under generation times of 30 and 
90 min (Bernstein et al., 2002). However, there was no available data for LpxA. We estimated the half life $\left(t_{1 / 2}\right)$ by taking the average half life from the available data of other mRNAs involved in the LPS biosynthesis. Thus, LpxA was assigned a half life of $3.3 \mathrm{~min}$ and the mean lifetime $\tau=t_{1 / 2} / \ln 2$ was estimated at $286 \mathrm{~s}$. This suggests that an mRNA would be degraded on average every $286 \mathrm{~s}$, hence Rate $_{\text {degm }}=1 / 286 s^{-1}$. It has been reported that the rate of protein translation is about 20 amino acids per second (Alberts et al., 2002) and would usually take about $2 \mathrm{~s}$ for ribosomes to bind to an mRNA (Mitarai et al., 2008). Given that LpxA consist of 262 amino acids (Coleman and Raetz, 1988), it would perhaps take $15 \mathrm{~s}$ for a protein to be translated. In addition, since the stability of an mRNA is in part due to its mean lifetime (Chen et al., 2008; Hoen et al., 2011), the model assumes the production of 19 proteins per mRNA lifetime. We therefore designated Rate trsl $_{\text {to }}$ to $19 / 286 \mathrm{~s}^{-1}$. On the other hand, $E$. coli proteins associated with the cell membranes usually possess half lives corresponding to the cell doubling time mainly from dilution resulting from cell division (Akiyama, 2009). Consequently, the half life of an LpxA protein was selected to be $30 \mathrm{~min}$ and the mean lifetime was estimated to be about $43 \mathrm{~min}$. Rate $\mathrm{degp}_{\mathrm{de}}$ was therefore assigned to be $1 / 2597 \mathrm{~s}^{-1}$.

\subsubsection{Estimation of Rate trans, $_{\text {Rate }}$ trsl, Rate $_{\text {degm }}$ and Rate degp $_{\text {in }}$ LpxC}

Due to the unfavourable equilibrium constant of LpxA, the model assumed more LpxC molecules might be required to act quickly on LpxA's product before it reverses back to the initial substrate. We therefore utilized a transcription burst number of 8 - just

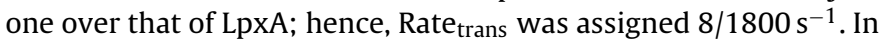
the absence of specific data as regards mRNA degradation rate for LpxC, we employed similar method utilized for LpxA by assigning a half-life of $3.3 \mathrm{~min}$ and subsequently a mean lifetime of $286 \mathrm{~s}$

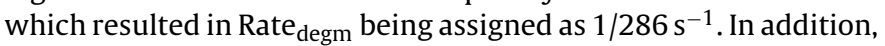
given that LpxC consist of 305 amino acids (Beall and Lutkenhaus, 1987), this suggests that a protein would be produced approximately every $17 \mathrm{~s}$ which approximates to 17 translations over the lifespan of an LpxC mRNA. The parameter Rate trsl $_{\text {was subsequently }}$ designated as $17 / 286 \mathrm{~s}^{-1}$. The abundance of LpxC is regulated via FtsH cleavage (Sorensen et al., 1996) which has a direct effect on the cell's generation time. In experiments conducted by Schäkermann et al. (2013), they reported half life values for LpxC presumably due to FtsH activity under conditions that results in different generation times. Their findings suggest that at high generation times, the half life of LpxC is low. From their reported data, we can estimate the half life of LpxC and the rate of FtsH cleavage under optimum conditions. However, there was no data at doubling time of $30 \mathrm{~min}$ at $37^{\circ} \mathrm{C}$. We therefore generated a non-linear regression curve of available data at $37^{\circ} \mathrm{C}$ and interpolated for unknown half-life at generation time of $30 \mathrm{~min}$ (Fig. 4A). Although, the experiments by Schäkermann et al. (2013) were carried out using two different culture media, each with its distinct generation time, this would have a negligible effect on our estimations because the half life of LpxC under FtsH activity is a direct function of the generation time; the higher the generation time, the smaller the half life. As a result, the half life of LpxC under optimum conditions and at a generation time of $30 \mathrm{~min}$ was estimated to be about $50 \mathrm{~min}$. Hence, our model

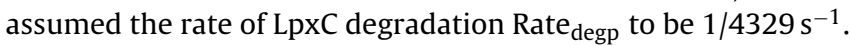

Eq. (1) proposed by Shahrezaei and Swain (2008) was reported to be accurate in comparison with experimental data whenever the ratio of $d_{0} / d_{1}$ is $\gg 1$ and is therefore is applicable to our model.

\subsection{Estimation of kinetic parameters for $L p x A$ and $L p x C$}

When $\mathrm{N}$-acetylglucosamine is used as substrate, the MichaelisMenten constant $K_{m}$ and turnover number $\left(k_{2}\right)$ of LpxA has been reported to be $0.82 \mathrm{mM}$ and $7.17 \mathrm{~s}^{-1}$ respectively (Wyckoff and
Raetz, 1999). We were therefore able to calculate the rate constant for LpxA binding to the substrate $\left(k_{1}\right)$, dissociation constant from the enzyme-substrate complex $\left(k_{-1}\right)$ and the reverse rate constant for the formation of complex from $k_{m}=\left(k_{-1}+k_{2}\right) / k_{1}$ and $k_{\text {eq }}=\left(k_{1} \times k_{2}\right) /\left(k_{-1} \times k_{-2}\right)$. We assumed the catalytic rate constant $\left(k_{2}\right)$ to be $1 \%$ the dissociation rate constant of the enzyme-substrate in order to favour the thermodynamically unfavourable equilibrium observed from experimental assays. Roberts et al. (2011) has shown that assumptions of this kind are negligible as long as the parameters $k_{-1}$ and $k_{1}$ correspond to the $K_{m}$ value. On the other hand, the $K_{m}$ and turnover number for LpxC whilst utilizing UDP3-O-(3-hydroxymyristoyl)-N-acetylglucosamine as substrate was reported to be $0.00019 \mathrm{mM}$ and $1.5 \mathrm{~s}^{-1}$ respectively (Hernick et al., 2005). Enzymic reactions in which the rate at which the product reverses back to substrate is much smaller than product synthesis rate, $k_{-2}$ is negligible.

\subsection{Estimation of amounts of substrate and enzymes}

It has been reported that there are approximately 1 million molecules of lipid A per E. coli cell (Raetz et al., 2009) and this probably is the concentration of lipid A just before the cell divides. The stoichiometric ratio of UDP-N-acetylglucosamine and UDP-3-O-(3hydroxymyristoyl) glucosamine is $1: 1$; indicating approximately 1 million molecules of initial substrate would be consumed by a single cell for the first two steps of the reaction. We therefore assigned UDP-N-acetylglucosamine an initial value of 1 million molecules. It is estimated that there are about 664 LpxA molecules per E. coli cell (Ishihama et al., 2008) and this probably would be the amount prior to cell division. From Eq. (1), we can estimate the number of proteins translated per generation time and therefore determine the initial concentration of LpxA. Additionally, the concentration of $\mathrm{LpxC}$ within an E. coli cell has not been experimentally determined. However, it is reasonable to make estimations from prior purification experimental data based on mass composition of the purified protein from cell lysates. When LpxC gene from Pseudomonas aeruginosa was cloned via a plasmid vector in an E. coli host (Hyland et al., 1997), it was reported to be overexpressed between 900 - and 1000-fold representing $3.7 \mathrm{mg}(14 \%$ yield) of $130 \mathrm{mg}$ of total protein in membrane-free crude lysate. We assumed that the protein was expressed 950 folds and were able to estimate that LpxC comprise of about $0.02 \%$ of cytoplasmic proteins given that there are about 1.9 million proteins present in the cytoplasm of E. coli (Sundararaj et al., 2004). This estimate was assumed to be the concentration of LpxC prior to cell division indicating that cleavage by FtsH has occurred. From information on amount of proteins synthesized as calculated from Eq. (1) and the rate of cleavage by FtsH, we can determine the initial amounts of LpxC at time $t=0$.

The model was run at a simulation time of 30 min using the COPASI software (Mendes et al., 2009) and the cytoplasmic compartment volume of E. coli was set at $6.7 \times 10^{-16} \mathrm{~L}$ (Cayley et al., 1991). The generated SBML file is found in the Supplementary Material section.

\section{Results}

\subsection{Estimation of number of LpxA and LpxC translated}

From Eq. (1), we were able to estimate the number of proteins produced over a period of $30 \mathrm{~min}$ by generation of a probability curve. The expression of LpxA was estimated to result in 75 proteins (Fig. 3A) while LpxC about 93 proteins (Fig. 3B). 

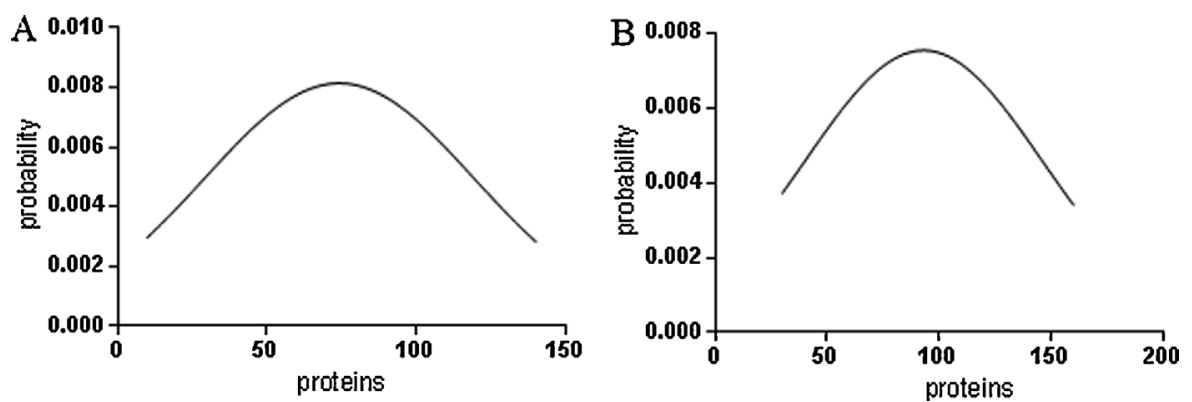

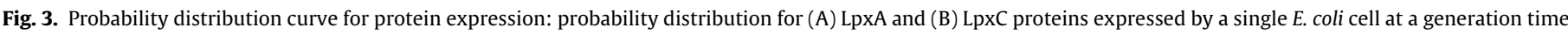
of $30 \mathrm{~min}$.

\subsection{Estimation of the half life of LpxC under FtsH conditions}

Schäkermann et al. (2013) reports that LpxC abundance is regulated by FtsH during slow growth conditions and from their data, we estimated the half life of $\mathrm{LpxC}$ at $37^{\circ} \mathrm{C}$ under a generation time of $30 \mathrm{~min}$ by interpolation for unknown half life from the generated non-linear regression curve (Fig. 4A). The half life of LpxC at $37^{\circ} \mathrm{C}$ under FtsH conditions was 50 min.

\subsection{Estimation of kinetic parameters for LpxA, LpxC and FtsH}

From the obtained $K_{m}$ and turnover values of LpxA and LpxC, we approximated the rate constants for enzyme binding to substrate $k_{1}$, enzyme-substrate complex dissociation constant $k_{2}$, and reverse rate constant for formation of the complex $k_{-2}$. For LpxA, we assumed the catalytic rate constant to be $1 \%$ the dissociation rate constant in favour of the thermodynamically unfavourable equilibrium constant $(\approx 0.01)$. This resulted in assigning values of $883 \mathrm{mM}^{-1} \mathrm{~s}^{-1}$ to $k_{1}, 717 \mathrm{~s}^{-1}$ and $883 \mathrm{mM}^{-1} \mathrm{~s}^{-1}$ to $K_{-1}$, and $k_{-2}$ respectively. Our model assumes approximately 664 molecules of LpxA per cell prior to cell division and given that about 75 molecules
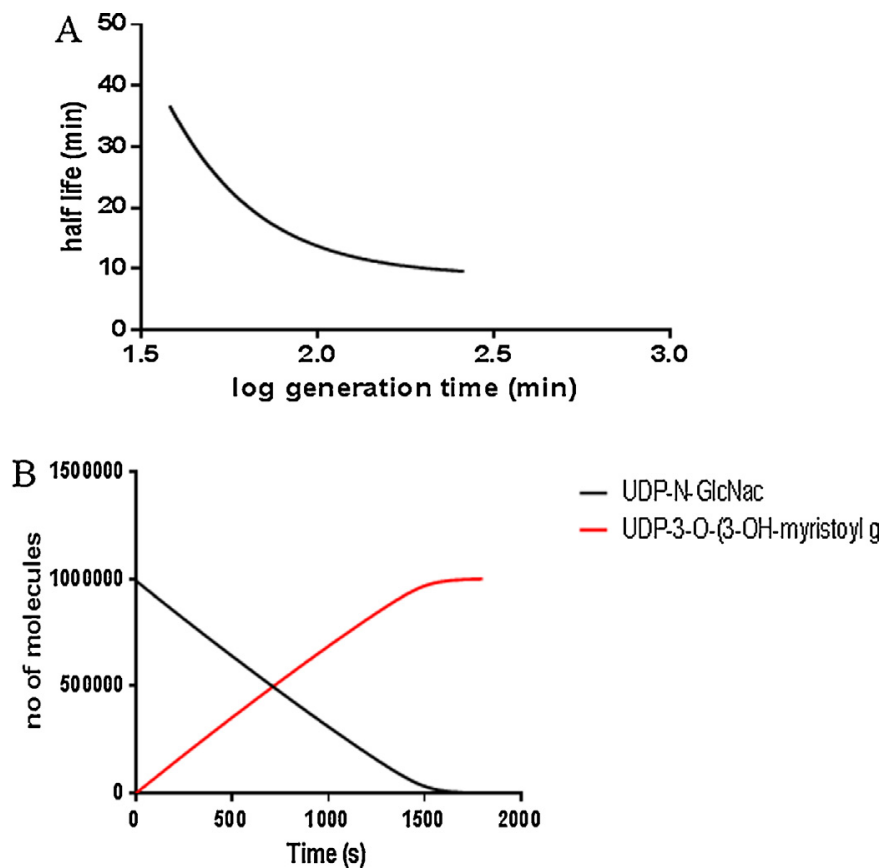

Fig.4. Catalytic efficiency of LpxC in the LPS pathway and its stability under different growth conditions. (A) Half life of $E$. coli LpxC under different generation times. (B) Formation of UDP-3-O-(3-hydroxymyristoyl)-glucosamine indicating that LpxC is sufficient to overturn the effect of the unfavourable equilibrium resulting from the first step of the LPS pathway. of LpxA would be produced over the course of $30 \mathrm{~min}$, the initial number of LpxA molecules at $t=0$ was set at 589 . From these estimates, we derived the rate constant from $\ln \left(E_{0} / E_{t}\right)=-k t$ and hence, production of LpxA over the course of simulation occurred with a rate constant of $7.0 \times 10^{-5} \mathrm{~s}^{-1}$. Similarly, the $k_{1}$ and $k_{-1}$ values of LpxC were $7973 \mathrm{mM}^{-1} \mathrm{~s}^{-1}$ and $0.015 \mathrm{~s}^{-1}$ respectively. Our model estimates about 385 molecules of LpxC prior to cell division and is assumed that proteolysis by FtsH has occurred at this stage. From the calculated half life of 50 min for LpxC under FtsH conditions at a generation time of $30 \mathrm{~min}$, we approximated the initial amount of LpxA at $t=0$ and the rate constant of cleavage from $N_{t}=N_{0}(1 / 2)^{t / t_{1 / 2}}$ and $\lambda=\ln 2 / t_{1 / 2}$. This resulted in a cleavage rate constant of $2.3 \times 10^{-4} \mathrm{~s}^{-1}$ and initial LpxC molecules of 583 . Nevertheless, without FtsH activity, the probable number of LpxC molecules after 30 min would probably remain 583 and given that we approximated the expression of 93 molecules over this time course, the initial number of LpxC molecules was set at 490 and the rate constant of translation was subsequently derived. However, since the number of cleaved LpxC molecules by FtsH is greater than the number of molecules translated over the simulation time course, the net rate constant for production of new molecules was in the negative, hence a value $-9.6 \times 10^{-5} \mathrm{~s}^{-1}$. This negative value was included for model fitting in order to attain about $385 \mathrm{LpxC}$ molecules after the simulation (Table 1 ).

\subsection{Simulation results}

The model was run at a simulation time of $30 \mathrm{~min}$ and the initial number of UDP-N-acetylglucosamine molecules was set at $10^{6}$ whilst setting initial values of LpxA and LpxC as 583 and 490 molecules respectively. After $30 \mathrm{~min}$, approximately all substrate molecules were converted to UDP-3-O-(3-hydroxymyristoyl) glucosamine (Fig. 4B) indicating that LpxC was sufficient to regularize the effect of the unfavourable equilibrium constant of the first reaction catalyzed by LpxA. We next investigated the proposed regulatory mechanism by $\mathrm{FtsH}$ protease and its capacity to normalize excessive production of end-product by cleavage of LpxC.

Table 1

Kinetic parameters used for simulation.

\begin{tabular}{lll}
\hline Reaction & Rate constants & Source $^{\mathrm{a}}$ \\
\hline $\mathrm{O} \rightarrow \mathrm{O}+$ LpxA & $0.00007 \mathrm{~s}^{-1}$ & $\mathrm{~K}$ \\
$\mathrm{LpxA}+\mathrm{S} \rightarrow$ ES & $883 \mathrm{mM}^{-1} \mathrm{~s}^{-1}$ & $\mathrm{~K}$ \\
$\mathrm{ES} \rightarrow$ LpxA + & $717 \mathrm{~s}^{-1}$ & $\mathrm{~K}$ \\
$\mathrm{ES} \rightarrow \mathrm{P}+\mathrm{LpxA}$ & $7.17 \mathrm{~s}^{-1}$ & $\mathrm{E}$ \\
$\mathrm{P}+\mathrm{LpxA} \rightarrow \mathrm{ES}$ & $883 \mathrm{mM}^{-1} \mathrm{~s}^{-1}$ & $\mathrm{~K}$ \\
$\mathrm{Q} \rightarrow \mathrm{Q}+$ LpxC & $0.000096 \mathrm{~s}^{-1}$ & $\mathrm{~K}$ \\
$\mathrm{LpxC}+\mathrm{P} \rightarrow$ EP & $7973 \mathrm{mM}^{-1} \mathrm{~s}^{-1}$ & $\mathrm{~K}$ \\
$\mathrm{EP} \rightarrow$ LpxC +P & $0.015 \mathrm{~s}^{-1}$ & $\mathrm{~K}$ \\
LpxC $\rightarrow$ degrade & $0.00023 \mathrm{~s}^{-1}$ & $\mathrm{~K}$ \\
$\mathrm{EP} \rightarrow$ P2 + LpxC & $1.5 \mathrm{~s}^{-1}$ & $\mathrm{E}$ \\
\hline
\end{tabular}

a $\mathrm{E}=$ experimental; $\mathrm{K}=$ from our kinetic model. 

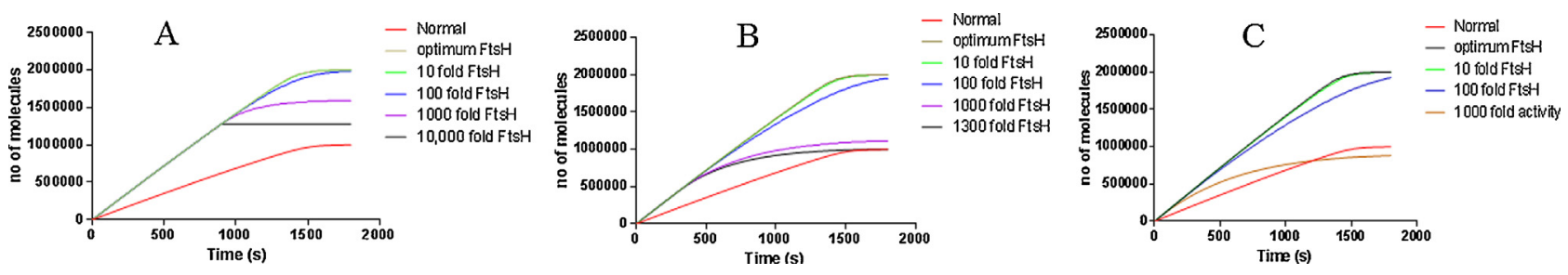

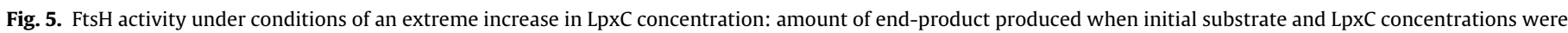

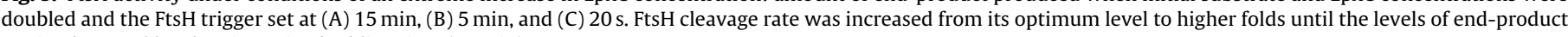
attained normal levels prior to its doubling time ( $30 \mathrm{~min}$ ).

In order to perform this analysis, we simulated a two-fold increase in LpxC molecules (Fig. 5). As a result, we also increased the number of initial substrate by two-folds in order to estimate the build up of excessive product under these conditions. Since there is neither experimental data nor appropriate means to estimate the exact concentration and exact time at which FtsH activity is triggered, simulations using differential trigger times were performed. We initially set the FtsH trigger at about $15 \mathrm{~min}$ (half doubling time) (Fig. 5A). This indicates that after $15 \mathrm{~min}$, FtsH would be activated to increase its rate of cleavage in order to attain the optimum end product after $30 \mathrm{~min}$. Under these conditions, FtsH was unable to regulate the formation of excess end-product even with an increased cleavage rate of 10,000 -folds. This could suggest that FtsH is activated at an earlier time. A second simulation was performed by setting the trigger time at about $5 \mathrm{~min}$ (Fig. 5B). However, under these conditions FtsH required an increase in cleavage rate by 1300 -fold in order to regulate the formation of end product to optimum. This again indicates that FtsH could be triggered at an earlier time. A third simulation was performed by setting the activation time at about $20 \mathrm{~s}$ (Fig. 5C). Again FtsH required a 1000-fold increase in cleavage rate in order to normalize product formation to optimal during the generation time of $30 \mathrm{~min}$. It is noteworthy that at this time of $20 \mathrm{~s}$, approximately $2.8 \%$ of the required optimal end product is formed and still required a 1000-fold increase in FtsH activity for regularization at the end of the simulation time. This may indicate that FtsH is either extremely sensitive to activation by downstream feedback or an alternative activation mechanism is being employed.

It is perfectly plausible that a two-fold increase in both substrate level and enzyme concentration is not physiologically likely under standard conditions but may well occur during protein overexpression or in cases involving cell division in which the enzyme pools have been asymmetrically distributed. In order to model what may occur under standard environmental conditions, we ran simulations in which the concentration of LpxC was gradually increased by 1.1,1.2- and 1.4-folds. Again, we set the trigger of FtsH at different time points of $15 \mathrm{~min}, 5 \mathrm{~min}$ and $20 \mathrm{~s}$. When LpxC amounts were increased by 1.4-fold and the trigger for increased FtsH activity set at $15 \mathrm{~min}$, the requirement of FtsH activity from its optimum was greater than a 1600 -fold to bring about an optimal production of end-product (Fig. 6A). Similarly, at an FtsH trigger time of $5 \mathrm{~min}$, a 600-fold increase in FtsH was required for optimal end product synthesis (Fig. 6B). However, just a 400 -fold increase in FtsH was sufficient for the regularization of end-product at the end of simulation at an activation time of $20 \mathrm{~s}$ (Fig. 6C). An increment in LpxC concentration by 1.2 -fold resulted in an increased cleavage requirement by FtsH of over 1000 -fold at an activation time of 15 min (Fig. 6D) and a 300-fold increase when activated at $5 \mathrm{~min}$ (Fig. 6E). However, at an earlier trigger time of 20 s, a $250-$ fold increase in proteolytic rate was required for regularization of
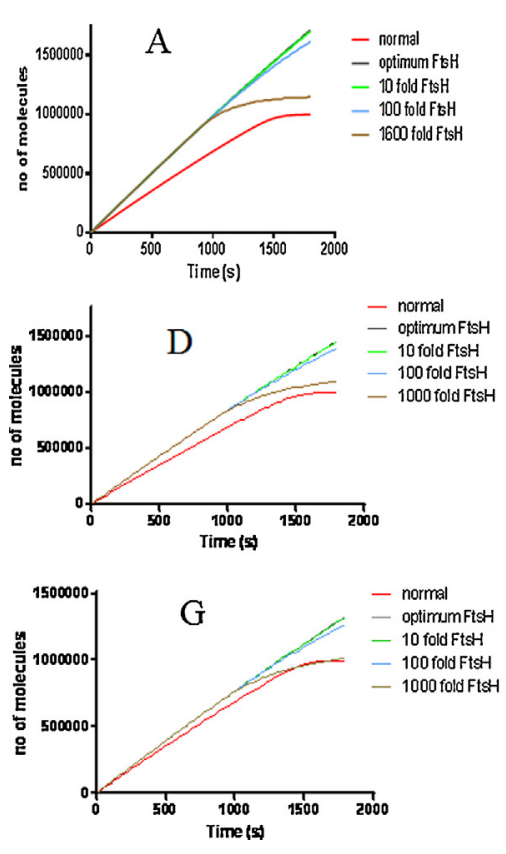
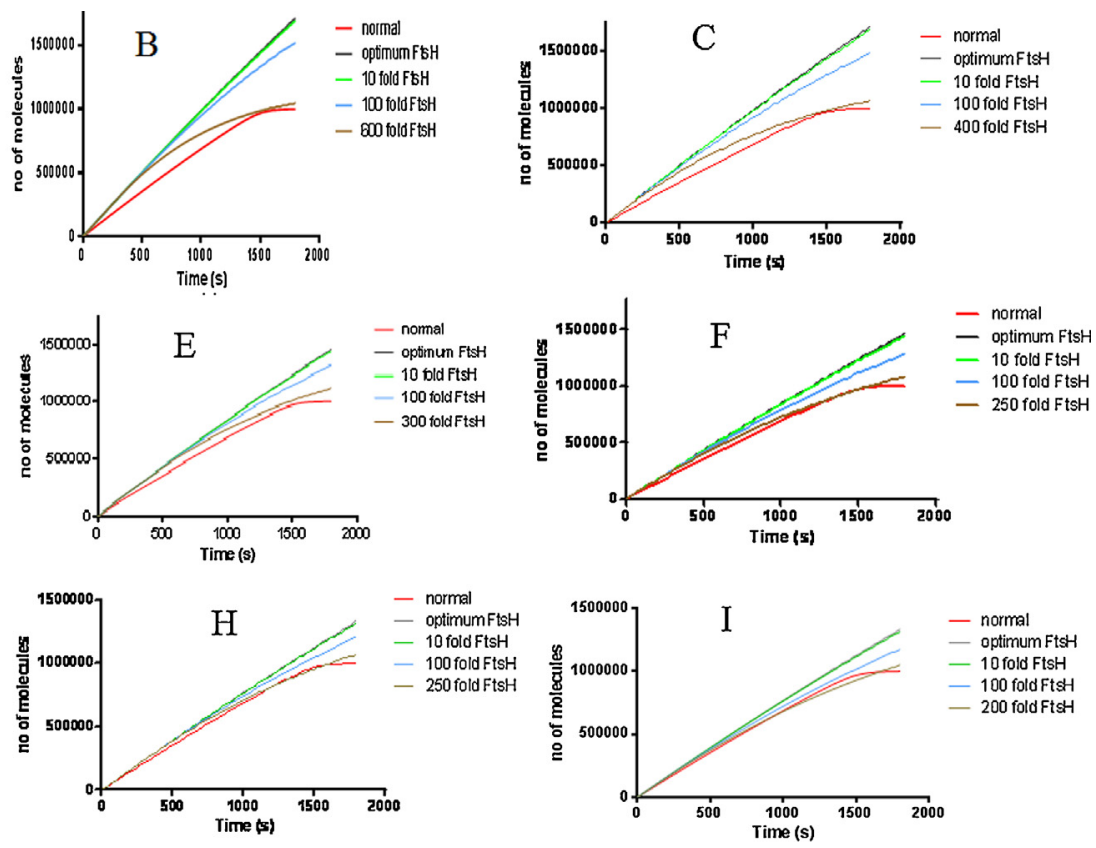

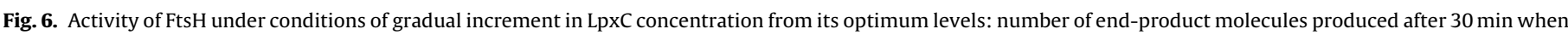

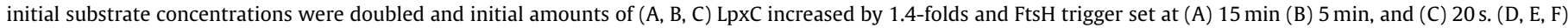

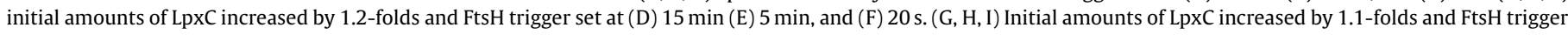
set at (G) $15 \mathrm{~min}(\mathrm{H}) 5 \mathrm{~min}$, and (I) $20 \mathrm{~s}$. 
end-product (Fig. 6F). The FtsH requirement at a 1.1-fold increase in LpxC amounts was much less. At a trigger point of $15 \mathrm{~min}$, an increased cleavage rate of 1000 -folds was required for normalization (Fig. 6G). Reducing the trigger time to $5 \mathrm{~min}$ reduced the rate requirement of FtsH to about 250-folds (Fig. 6H). A further trigger reduction time to $20 \mathrm{~s}$ resulted in an increased cleavage requirement rate of 200 -folds (Fig. 6I). At this trigger point, about $1.4 \%$ of the required optimal end-product is made, yet required a 200 -fold increased proteolytic activity to bring down the levels of end-product to optimum.

\section{Discussion}

Despite its obvious biological significance, biosynthesis of Lipid $A$ and importantly its regulatory mechanisms are poorly understood. The complete Lipid A pathway in E. coli consists of nine enzymes in length and involves nine intermediates (Raetz et al., 2007). It is not surprising perhaps that experimental work is incomplete and whilst this forms a very useful framework, it is unable to generate predictions involving the behaviour of the whole pathway under variable conditions. Such information would be invaluable for the fields of bacteriology, immunology and of course drug discovery.

Experimental research has identified LpxA as the first step in the LPS biosynthetic pathway and due to its unfavourable equilibrium constant (Anderson et al., 1993), LpxC is thought to be the first committed enzyme (Barb and Zhou, 2008). No experimental data are available to clarify whether such a large unfavourable equilibrium constant could be capable of providing the number of intermediary metabolites required for the synthesis of LPS. It has been postulated that reactions with such highly unfavourable equilibrium constant may not actually exist in vivo (Lunn et al., 1990). However, our simulations (using known kinetic parameters for LpxA and LpxC) suggest that the catalytic activity of LpxC is indeed sufficient to overturn the unfavourable equilibrium constant proposed for LpxA (Fig. 4B). This analysis confirms experimental observations that LpxC represents the first committed step in LPS biosynthesis. Additionally, our model suggests that the rate at which LpxC forms a complex with its substrate is nine-fold greater than the rate at which LpxA catalyzes the reverse reaction.

Recent studies have identified FtsH as being an important regulatory protease involved in the LPS biosynthetic pathway (Langklotz et al., 2011). This protease is thought to modulate levels of LpxC by a cleavage mechanism following its own activation by an unknown metabolite. Unfortunately, there are no experimentally derived kinetic parameters for this protease. However, we were able to establish a proteolytic rate by regression analysis from a recent study Schäkermann et al. (2013). Using these parameters, we investigated the capacity of FtsH to normalize product formation under two environmental conditions; extreme increment in LpxC levels (as may occur during protein over-expression) and moderate increases in LpxC concentrations.

Under extreme LpxC increments, the simulations indicated that $\mathrm{FtsH}$ is required to be activated very early following increased metabolic activity and furthermore, that its cleavage rate was required to be increased in the 10,000 -folds in order to lower the concentration of the build up of toxic metabolites. However, this increased rate was still insufficient to lower the concentration of end-product to its optimal concentrations. This analysis implies that there may be alternative and/or additional mechanisms regulating LPS synthesis under these extreme conditions. Under slight increment in LpxC amounts, we found that increased activity of FtsH by 200-fold (Fig. 6I) was sufficient to return the output of the pathway to normal levels of metabolites. Whilst this is within the limits of other known allosterically activiated enzyme activities
(Hardie, 2011), it has not been established experimentally for FtsH. Under such modest increases in LpxC concentrations, only a small percentage of the optimal end product is formed at the point of FtsH activation which implies that this protease is either extremely sensitive to its activator or that the cleavage of LpxC plays only a minor role in the regulatory process of LPS production. It is known that FtsH also cleaves a secondary enzyme within this pathway (KDtA) (Katz and Ron, 2008), whilst other enzymes are also under differing regulatory mechanisms. However, the fact that LpxC is cleaved points to some important regulatory process. We have established that cleavage of LpxC will result in lowering the flow or flux of substrate into LPS synthesis, as LpxA activity is highly unfavourable. Therefore, any reduction in LpxC will result in a larger pool of initial substrate which the cell can use for other metabolic processes such as peptidoglycan biosynthesis (Anderson and Raetz, 1987). An additional reason for the cleavage of LpxC could be in cases of asymmetrical cell division. When the bacterial cell divides, usually each daughter cell gets about half the amount of components from the parent cell. However, it has been reported (Ventura and Sourjik, 2011) that some daughter cells could receive more than the required amounts, while others receive less. Again, a quick activation of FtsH could be vital in regulating substrate flow in instances where the cell has excess amount of LpxC immediately after cell division. We therefore, propose that LpxC cleavage by Fts $\mathrm{H}$ is important in order to switch the flow of the pathway under slow growth conditions and additionally to modulate enzyme levels following cell division and not necessarily to regulate the levels of LPS.

In order to clarify the role played by FtsH during LPS metabolism we are currently constructing and conducting simulations involving the entire pathway along with their regulatory components. By conducting such studies it may be possible to identify the metabolite which is most likely to trigger the activation of $\mathrm{FtsH}$ and importantly uncover the main regulatory processes contributing to the synthesis of this biologically important molecule.

\section{Appendix A. Supplementary data}

Supplementary data associated with this article can be found, in the online version, at http://dx.doi.org/10.1016/j.compbiolchem. 2013.06.001.

\section{References}

Akiyama, Y., 2009. Quality control of cytoplasmic membrane proteins in Escherichic coli. J. Biochem. 146, 449-454.

Alberts, B., Johnson, A., Lewis, J., Raff, M., Roberts, K., Walter, P., 2002. Molecula Biology of the Cell, fourth ed. Garland Science, New York.

Anderson, M.S., Bulawa, C.E., Raetz, C.R., 1985. The biosynthesis of gram-negative endotoxin. Formation of lipid A precursors from UDP-GlcNAc in extracts of Escherichia coli. J. Biol. Chem. 260, 15536-15541.

Anderson, M.S., Bull, H.G., Galloway, S.M., Kelly, T.M., Mohan, S., Radika, K., Raetz, C.R., 1993. UDP-N-acetylglucosamine acyltransferase of Escherichia coli. The first step of endotoxin biosynthesis is thermodynamically unfavourable. J. Biol Chem. 268, 19858-19865.

Anderson, M.S., Raetz, C.R., 1987. Biosynthesis of lipid A precursors in Escherichia coli. A cytoplasmic acyltransferase that converts UDP-N-acetylglucosamine to UDP-3-O-(R-3-hydroxymyristoyl)-N-acetylglucosamine. J Biol. Chem. 262, 5159-5169.

Barb, A.W., Zhou, P., 2008. Mechanism and inhibition of LpxC: an essential zincdependent deacetylase of bacterial lipid A synthesis. Curr. Pharm. Biotechnol. 9 9-15.

Beall, B., Lutkenhaus, J., 1987. Sequence analysis, transcriptional organization, and insertional mutagenesis of the envA gene of Escherichia coli. J. Bacteriol. 169, 5408-5415.

Belunis, C.J., Raetz, C.R., 1992. Biosynthesis of endotoxins. Purification and catalytic properties of 3-deoxy-D-manno-octulosonic acid transferase from Escherichia coli. J. Biol. Chem. 267, 9988-9997.

Bernstein, J.A., Khodursky, A.B., Lin-Chao, P.L.S., Cohen, S.N., 2002. Global analysis of mRNA decay and abundance in Escherichia coli at single-gene resolution using two-color fluorescent DNA microarrays. Proc. Natl. Acad. Sci. U.S.A. 99 9697-9702. 
Cayley, S., Lewis, B.A., Guttman, H.J., Record Jr., M.T., 1991. Characterization of the cytoplasm of Escherichia coli K-12 as a function of external osmolarity. Implications for protein-DNA interactions in vivo. J. Mol. Biol. 222, 281-300.

Chen, C.Y., Ezzeddine, N., Shyu, A.B., 2008. Messenger RNA half-life measurements in mammalian cells. Methods Enzymol. 448, 335-357.

Coleman, J., Raetz, C.R.H., 1988. First committed step of lipid A biosynthesis in Escherichia coli: sequence of the lpxA gene. J. Bacteriol. 170, 1268-1274.

Creuzenet, C., Belanger, M., Wakarchuk, W.W., Lam, J.S., 2000. Expression, purification, and biochemical characterization of WbpP, a new UDP-GlcNAc C4 epimerase from Pseudomonas aeruginosa serotype O6. J Biol. Chem. 275, 19060-19067.

Garrett, T.A., Kadrmas, J.L., Raetz, C.R., 1997. Identification of the gene encoding the Escherichia coli lipid A 4'-kinase. Facile phosphorylation of endotoxin analogs with recombinant LpxK. J. Biol. Chem. 272, 21855-21864.

Golding, I., Paulsson, J., Zawilski, S.M., Cox, E.C., 2005. Real-time kinetics of gene activity in individual bacteria. Cell 123, 1025-1036.

Hardie, D.G., 2011. AMP-activated protein kinase: an energy sensor that regulates all aspects of cell function. Genes Dev. 25, 1895-1908.

Hernick, M., Gennadios, H.A., Whittington, D.A., Rusche, K.M., Christianson, D.W., Fierke, C.A., 2005. UDP-3-O-((R)-3-hydroxymyristoyl)-N-acetylglucosamine deacetylase functions through a general acid-base catalyst pair mechanism. J. Biol. Chem. 280, 16969-16978.

Hoen, P.A., Hirsch, M., de Meijer, E.J., de Menezes, R.X., van Ommen, G.J., den Dunnen, J.T., 2011. mRNA degradation controls differentiation state-dependent differences in transcript and splice variant abundance. Nucleic Acids Res. 39, 556-566.

Hyland, S.A., Eveland, S.S., Anderson, M.S., 1997. The lipid A biosynthesis pathway. Pseudomonas aeruginosa: a metalloamidase of UDP-3-O-acyl-GlcNAc deacetylase from cloning. J. Bacteriol. 179, 2029-2037.

Ishihama, Y., Schmidt, T., Rappsilber, J., Mann, M., Hart, F.U., Kerner, M.J., Frishman, D., 2008. Protein abundance profiling of the Escherichia coli cytosol. BMC Genomics 9, http://dx.doi.org/10.1186/1471-2164-9-102.

Katz, C., Ron, E.Z., 2008. Dual role of FtsH in regulating lipopolysaccharide biosynthesis in Escherichia coli. J. Bacteriol. 190, 7117-7122.

Langklotz, S., Schäkermann, M., Narberhaus, F., 2011. Control of lipopolysaccharide biosynthesis by FtsH-mediated proteolysis of LpxC is conserved in enterobacteria but not in all gram-negative bacteria. J. Bacteriol. 193, 1090-1097.

Lunn, J.E., Droux, M., Martin, J., Douce, R., 1990. Localization of ATP Sulfurylase and O-acetylserine(thiol)lyase in spinach leaves. Plant Physiol. 94, 1345-1352.

Mackie, G.A., 2013. RNase E: at the interface of bacterial RNA processing and decay. Nat. Rev. Microbiol. 11, 45-57.

McAdams, H.H., Arkin, A., 1997. Stochastic mechanisms in gene expression. Proc. Natl. Acad. Sci. U.S.A. 94, 814-819.

Mendes, P., Hoops, S., Sahle, S., Gauges, R., Dada, J., Kummer, U., 2009. Computational modelling of biochemical networks using COPASI. Methods Mol. Biol. 500, 17-59.

Mitarai, N., Sneppen, K., Pedersen, S., 2008. Ribosome collisions and translation efficiency: optimization by codon usage and mRNA destabilization. J. Mol. Biol. 382, $236-245$.
Ogura, T., Inoue, K., Tatsuta, T., Suzaki, T., Karata, K., Young, K., Su, L.H., Fierke, C.A., Jackman, J.E., Raetz, C.R., Coleman, J., Tomoyasu, T., Matsuzawa, H., 1999. Balanced biosynthesis of major membrane components through regulated degradation of the committed enzyme of lipid A biosynthesis by the AAA protease FtsH (HflB) in Escherichia coli. Mol. Microbiol. 31, 833-844.

Plank, L.D., Harvey, J.D., 1979. Generation Time Statistics of Escherichia coli B measured by synchronous culture techniques. J. Gen. Microbiol. 115, 69-77.

Poxton, I.R., 1995. Antibodies to Lipopolysaccharide. J. Immunol. Methods 186, 1-15.

Raetz, C.R., Whitfield, C., 2002. Lipopolysaccharide endotoxins. Annu. Rev. Biochem. 71, 635-700.

Raetz, C.R., Reynolds, C.M., Trent, M.S., Bishop, R.E., 2007. Lipid A modification systems in gram-negative bacteria. Annu. Rev. Biochem. 76, 295-329.

Raetz, C.R., Guan, Z., Ingram, B.O., Six, D.A., Song, F., Wang, X., Zhao, J., 2009. Discovery of new biosynthetic pathways: the lipid A story. J. Lipid Res. 50, S103-S108.

Roberts, E., Magis, A., Ortiz, J.O., Baumeister, W., Luthey-Schulten, Z., 2011. Noise contributions in an inducible genetic switch: a whole-cell simulation study. PLoS Comput. Biol. 7, http://dx.doi.org/10.1371/journal.pcbi.1002010.

Schäkermann, M., Langklotz, S., Narberhaus, F., 2013. FtsH-mediated coordination of lipopolysaccharide biosynthesis in Escherichia coli correlates with the growth rate and the alarmone (p)ppGpp. J. Bacteriol. http://dx.doi.org/10.1128/JB.02134-12.

Shahrezaei, V., Swain, P.S., 2008. Analytical distributions for stochastic gene expression. Proc. Natl. Acad. Sci. U.S.A. 105, 17256-17261.

Shih, Y., Whitesides, G.M., 1977. Large-scale ATP-requiring enzymatic phosphorylation of creatine can be driven by enzymatic ATP regeneration. J. Org. Chem. 42, 4165-4166.

Sorensen, P.G., Lutkenhaus, J., Young, K., Eveland, S.S., Anderson, M.S., Raetz, C.R. 1996. Regulation of UDP-3-O-[R-3-hydroxymyristoyl]-N-acetylglucosamine deacetylase in Escherichia coli. The second enzymatic step of lipid a biosynthesis. J. Biol. Chem. 271, 25898-25905.

Sundararaj, S., Guo, A., Habibi-Nazhad, B., Rouani, M., Stothard, P., Ellison, M. Wishart, D.S., 2004. The CyberCell Database (CCDB): a comprehensive, selfupdating, relational database to coordinate and facilitate in silico modeling of Escherichia coli. Nucleic Acids Res. 32, D293-D295.

Ventura, B.D., Sourjik, V., 2011. Self-organized partitioning of dynamically localized proteins in bacterial cell division. Mol. Syst. Biol. 7, http://dx.doi.org/10.1038/msb.2010.111.

Walker, S.L., Redman, J.A., Elimelech, M., 2004. Role of cell surface lipopolysaccharides in Escherichia coli K12 adhesion and transport. Langmuir 20, 7736-7746

Wyckoff, T.J.O., Raetz, C.R.H., 1999. The active site of Escherichia coli UDP-Nacetylglucosamine acyltransferase. Chemical modification and site-directed mutagenesis. J. Biol. Chem. 274, 27047-27055.

Young, K., Silver, L.L., Bramhill, D., Cameron, P., Eveland, S.S., Raetz, C.R., Hyland, S.A., Anderson, M.S., 1995. The envA permeability/cell division gene of Escherichia coli encodes the second enzyme of lipid A biosynthesis. UDP-3-O(R-3-hydroxymyristoyl)-N-acetylglucosamine deacetylase. J. Biol. Chem. 270, 30384-30391. 